\title{
Repeat transcatheter aortic valve implantation using a latest generation balloon-expandable device for treatment of failing transcatheter heart valves
}

Andreas Schaefer ${ }^{1 *}$, Hendrik Treede ${ }^{1}$, Moritz Seiffert ${ }^{2}$, Florian Deuschl $^{2}$, Niklas Schofer ${ }^{2}$, Yvonne Schneeberger $^{1}$, Stefan Blankenberg ${ }^{2}$, Hermann Reichenspurner ${ }^{1}$, Ulrich Schaefer ${ }^{2}$ and Lenard Conradi ${ }^{1}$

\begin{abstract}
Background: Paravalvular leakage (PVL) is a known complication of transcatheter aortic valve implantation (TAVI) and is associated with poor outcome. Besides balloon-post-dilatation, valve-in-valve (ViV) procedures can be taken into consideration to control this complication. Herein we present initial experience with use of the latest generation balloon-expandable Edwards Sapien $3^{\circledR}$ (S3) transcatheter heart valve (THV) for treatment of failing THVs.

Methods: Between 01/2014 and 12/2014 three patients (two male, age: 71-80 y, log EUROScore I: 11.89 - 32.63) with failing THVs were refered to our institution for further treatment. THV approach with secondary implantation of an S3 was chosen after mutual agreement of the local interdisciplinary heart team at an interval of 533-1119 days from the index procedure. The performed procedures consisted of: S3 in Sapien XT, JenaValve and CoreValve.

Results: Successful transfemoral implantation with significant reduction of PVL was achieved in all cases. No intraprocedural complications occured regarding placement of the $\mathrm{S} 3$ with a postprocedural effective orifice area (EOA) of $1.5-2.5 \mathrm{~cm}^{2}$ and pressure gradients of max/mean $14 / 6-36 / 16 \mathrm{mmHg}$. 30-day mortality was $0 \%$. At the latest follow-up of 90-530 days, all patients are alive and well with satisfactory THV function. Regarding VARC-2 criteria one major bleeding and one TIA was reported.
\end{abstract}

Conclusions: In the instance of moderate or severe aortic regurgitation after TAVI, S3 ViV deployment is an excellent option to reduce residual regurgitation to none or mild. For further assertions concerning functional outcomes long-term results have to be awaited.

Keywords: Aortic valve disease, Aortic valve stenosis, Paravalvular leak, Percutaneous valve therapy, Structural heart disease intervention, TAVI, THV

\section{Background}

Transcatheter aortic valve implantation (TAVI) has found widespread acceptance as alternative to surgical aortic valve replacement (SAVR) over the last decade. Through introduction of transcatheter heart valves (THV) into clinical daily routine, mortality of inoperable or high-risk patients was significantly reduced compared to medical therapy $[1,2]$. In 2012, the PARTNER trial presented data

\footnotetext{
* Correspondence: and.schaefer@uke.de

'Department of Cardiovascular Surgery, University Heart Center Hamburg,

Martinistraße 52, D-20246 Hamburg, Germany

Full list of author information is available at the end of the article
}

which showed comparable results of the first generation Edwards Sapien THV (Edwards Lifesciences Co., Irvine, CA, USA) and SAVR for severe aortic stenosis in high-risk patients regarding mortality, reduction in symptoms and valve hemodynamics [3]. Despite introduction of a wide variety of THVs into the medical sector, with largely favorable results $[4,5]$ and improved delivery systems, imaging or access site techniques [6-8], one major problem of THVs remains paravalvular leakage (PVL). PVL has a significantly higher incidence following TAVI compared to SAVR and leads to increased mortality if grade of PVL is $\geq$ moderate (2-year mortality of $30 \%$ in mild aortic 
regurgitation, $40 \%$ in moderate, 3-year mortality of $60 \%$ in severe aortic regurgitation) [9, 10]. For failing THVs, different strategies had to be developed to control these complications, avoid acute hemodynamic compromise and preserve therapeutic benefits of the TAVI procedure. Besides balloon-post-dilatation, valve-in-valve (ViV) procedures can be taken into consideration $[11,12]$. It was shown for the Edwards Sapien XT THV, that it is suitable for ViV procedures in cases of failing CoreValve THV (Medtronic Ltd., Dublin, IRL) with relevant PVL [13]. To date only one case report exists regarding suitability of the Edwards Sapien 3 (S3) THV for ViV treatment of a failing CoreValve THV [14]. In this case series, we report initial experience with the S3 for ViV therapy of different failing THVs in cases of relevant residual PVL.

\section{Methods}

\section{Preprocedural diagnostics}

Routine preprocedural diagnostic work-up included transthoracic (TTE) and transesophageal echocardiography (TEE), coronary angiogram, as well as contrast-enhanced, electrocardiogram-gated multislice computed tomography (CT) scans. Decision for ViV procedure was made after mutual agreement of the local interdisciplinary heart team. All described procedures were performed in a hybrid operating room by an interdisciplinary team consisting of cardiac surgeons and interventional cardiologists.

\section{Patients}

Three procedures were performed using the S3 THV for failing THV between $01 / 2014$ and $12 / 2014$. All patients presented with prohibitive operative risk for SAVR with a mean logistic European System for Cardiac Operative Risk Evaluation I (log EuroSCORE) of 11.89 - 32.63 and $\log$ EuroSCORE II of 2.33-19.14. Two of them were male and rang of age was 71-80 years. In one patient scheduled $\mathrm{ViV}$ procedure was the third interventional cardiac procedure with a status post CoreValve and Mitraclip implantation. All patients suffered from atrial fibrillation. Detailed clinical data are summarized in Table 1. All procedures which were carried out are in compliance with the Helsinki Declaration.

Table 1 Clinical baseline data and initial TAVI procedure information

\begin{tabular}{|c|c|c|c|}
\hline & Patient no. 1 & Patient no. 2 & Patient no. 3 \\
\hline Age (y) & 80 & 78 & 71 \\
\hline Gender & female & male & male \\
\hline BMI $\left(\mathrm{kg} / \mathrm{m}^{2}\right)$ & 46.6 & 21 & 21.6 \\
\hline Comorbidities & $\begin{array}{l}\text { AF, RI, osteoporosis, s/p TKA both } \\
\text { sides, AH, NIDDM }\end{array}$ & $\begin{array}{l}A F, 1 \text { VD, prostate cancer, s/p rectum } \\
\text { cancer, COPD IV, AH }\end{array}$ & $\begin{array}{l}\mathrm{s} / \mathrm{p} \text { DES in } L A D, 3 V D, s / p \\
\text { Mitraclip, AF }\end{array}$ \\
\hline logEuroSCORE I (\%) & 23.92 & 11.89 & 32.63 \\
\hline logEuroSCORE II (\%) & 5.54 & 2.33 & 19.14 \\
\hline STS PROM (\%) & 4.69 & 3.78 & 2.744 \\
\hline NYHA & III & III & IV \\
\hline \multicolumn{4}{|l|}{ TTE before index procedure } \\
\hline LVEF (\%) & 60 & 50 & 30 \\
\hline $\mathrm{EOA}\left(\mathrm{cm}^{2}\right)$ & 0.9 & 0.7 & 0.9 \\
\hline Gradient max/mean (mmHg) & $82 / 41$ & $90 / 52$ & $30 / 12$ \\
\hline Valve insufficiency & none & none & none \\
\hline \multicolumn{4}{|l|}{ Index procedure } \\
\hline Implanted THV, size (mm) & Edwards Sapien XT, 26 & JenaValve, 27 & CoreValve, 29 \\
\hline Symptoms after index procedure & syncope, dyspnea & dyspnea & dyspnea \\
\hline Time to $2^{\text {nd }}$ procedure $(\mathrm{d})$ & 1119 & 533 & 814 \\
\hline \multicolumn{4}{|l|}{ TTE after index procedure } \\
\hline $\mathrm{EOA}\left(\mathrm{cm}^{2}\right)$ & / & 1.9 & 2.8 \\
\hline Gradient max/mean (mm Hg) & / & $27 / 10$ & $15 / 9$ \\
\hline Valve insufficiency & mild & mild & mild \\
\hline
\end{tabular}

$Y$ years; BMI body mass index; logEuroSCORE logistic European System for Cardiac Operative Risk Evaluation; STS PROM Society of Thoracic Surgeons Predicted Risk of Mortality; NYHA New York Heart Association; TTE transthoracic echocardiography; LVEF left ventricular ejection fraction; EOA effective orifice area; TAVI transcatheter aortic valve implantation; THV transcatheter heart valve; $A F$ atrial fibrillation; $R /$ renal insufficiency; $T K A$ total knee arthroplasty; $A H$ arterial hypertension; NIDDM non insulin dependent diabetes mellitus; VD vessel disease; $s / p$ status post; COPD chronical obstructive pulmonal disease; PVL paravalvular leakage; SD standard deviation 


\section{Initial TAVI procedure}

Initial TAVI procedures were performed via transfemoral (tf) access in two cases (Edwards Sapien XT, CoreValve) and via transapical (ta) access in one case (JenaValve). Implantation was performed due to severe aortic stenosis in all cases. TTE prior to discharge after index procedure presented mild PVL in all cases with sufficient EOA and pressure gradients and patients were discharged with improved NYHA functional class of $\leq$ II. Index procedural data are summarized in Table 1.

At an interval of 533-1119 days, patients were readmitted with acute exacerbation of dyspnea or status post syncope. In patient 1 serial TTE examination due to recurrent cardiac decompensation presented severe PVL from one year after the index procedure, which was reluctantly observed and re-intervention was refused due to the high-risk profile. In the other two patients first detection of moderate or severe PVL of the THV led to immediate reinterventions. Severe PVL was demonstrated in all patients on TTE at time of admission. Detailed echocardiography data before ViV procedure are shown in Table 2.

\section{Valve-in-Valve implantation procedure}

Due to echocardiographic findings and reduced clinical status in all three cases, repeat TAVI was indicated following heart team consensus. Sizing was conducted by reference to $\mathrm{CT}$ of the index procedures to reduce radiation exposure with effective annulus sizes (based on $C \mathrm{~T}_{\text {eff }}=2 \times \sqrt{ }($ circumferential area/ $\pi)$ of $23 \mathrm{~mm}$ for the Sapien XT patient, $29 \mathrm{~mm}$ for the JenaValve patient and $24 \mathrm{~mm}$ for the CoreValve patient.

Due to adequate peripheral vasculature as assessed by reconstruction of the planning $\mathrm{CT}$, all procedures were performed via percutaneous $\mathrm{tf}$ access following standard procedural protocol. After insertion of a vascular closure system (ProStar XL Percutaneous Vascular Surgical System $^{\circ}$, Abbott Vascular, Santa Clara, CA, USA) in the right femoral artery and placement of a pacemaker lead in the RV via the right jugular vein, a guide wire was advanced retrogradely across the THV into the LV. Subsequently the S3 THV was deployed into the THV in a phase of rapid ventricular pacing, with prior positioning of the radio-opaque marker at the height of native the aortic annulus. Intraprocedurally, TEE and aortic root angiography as well as invasive pressure measurements were performed to document adequate THV function.

In two cases a cerebral protection system (Claret Medical, Inc., Santa Rosa, CA, USA) was placed via the right brachial artery into the brachiocephalic trunk and left common carotid artery to protect against possible distal embolization. Intraprocedural data are summarized in Table 2.

Events were recorded according to Valve Academic Research Consortium 2 (VARC-2) standard criteria.

Table 2 Preprocedural and postprocedural echocardiography data, Intraprocedural information

\begin{tabular}{|c|c|c|c|}
\hline & Patient no. 1 & Patient no. 2 & Patient no. 3 \\
\hline \multicolumn{4}{|l|}{ TTE/TEE before $2^{\text {nd }}$ procedure } \\
\hline LVEF (\%) & 60 & 50 & 30 \\
\hline Gradient max/mean (mm Hg) & $18 / 9$ & $24 / 12$ & $15 / 12$ \\
\hline $\mathrm{EOA}\left(\mathrm{cm}^{2}\right)$ & 1.7 & 2.2 & 2.3 \\
\hline Valve insufficiency & severe & moderate-severe & severe \\
\hline ViV procedure & Edwards Sapien XT & JenaValve & CoreValve \\
\hline Edwards Sapien 3 size $(\mathrm{mm})$ & 26 & 29 & 29 \\
\hline Procedure time $(\mathrm{mm})$ & 95 & 100 & 180 \\
\hline Fluoroscopy time (min) & 10 & 11 & 20 \\
\hline Contrast agent $(\mathrm{mL})$ & 121 & 125 & 174 \\
\hline \multicolumn{4}{|l|}{ TTE discharge } \\
\hline LVEF (\%) & 60 & 50 & 30 \\
\hline $\mathrm{EOA}\left(\mathrm{cm}^{2}\right)$ & 1.5 & 2.5 & 2.5 \\
\hline Gradient max/mean (mm Hg) & $36 / 16$ & $17 / 9$ & $14 / 6$ \\
\hline Paravalvular leakage & none & trace & trace \\
\hline ICU stay $(d)$ & 2 & 1 & 1 \\
\hline Hospital stay $(\mathrm{d})$ & 10 & 8 & 20 \\
\hline VARC -2 events at 30 days & Major bleeding & None & $\mathrm{TIA}$ \\
\hline
\end{tabular}

TTE transthoracic echocardiography; TEE transesophageal echocardiography; LVEF left ventricular ejection fraction; ViV valve-in-valve; $m m$ millimeter; min minutes; $\mathrm{mL}$ milliliter; EOA effective orifice area; ICU intensive care unit; VARC-2 Valve academic research consortium; TIA transitory ischemic attack; SD standard deviation 


\section{Results}

All patients were successfully implanted with a S3 THV for PVL of previous implanted THV without major complications such as stroke, myocardial infarction due to coronary obstruction or significant aortic regurgitation. Immediate intraprocedural invasive mean pressure measurements showed decline of $10 \mathrm{mmHg}$ to $5 \mathrm{mmHg}$ for S3 in Sapien XT, $10 \mathrm{mmHg}$ to $4 \mathrm{mmHg}$ for S3 in Jenavalve and $21 \mathrm{mmHg}$ to $12 \mathrm{mmHg}$ for $\mathrm{S} 3$ in CoreValve. In all patients severity of PVL was reduced to none/trace as documented by intraprocedural TEE, angiography and invasive measurements of hemodynamics. Findings were confirmed by TTE at the time of discharge. Echocardiography results are summarized in Table 2 .

Figure 1 shows development of EOA, PVL grade and max/mean pressure gradient over time from initial evaluation until discharge after second procedure. One patient showed slightly decrease of EOA without resulting in clinical symptoms such as dyspnea after the procedure. In all other patients increase in EOA was achieved. Accordingly in TTE prior to discharge mean pressure gradients were lowered in two patients but peak pressure gradients increased in two patients.

Intraprocedural angiographies are depicted in Fig. 2.

Three patients presented PVL two to three years after the index procedure and were implanted with an S3 electively with a hospital stay of $8-20 \mathrm{~d}$ and an intensive care unit stay of $1-2 \mathrm{~d}$.

According to VARC-2 criteria one major bleeding (access site hematoma requiring transfusion of two units of red blood cells) and one transient ischemic attack (TIA) was observed. TIA became apparent in sensory loss of the right hand of a patient with pre-existing severe stenosis of the left internal carotid artery. Symptoms subsided after $12 \mathrm{~h}$. None of the patients had acute kidney injury or need of renal replacement therapy. All patients were alive at 30 days of follow-up and at the time of 90 530 days after the procedure, respectively.

\section{Discussion}

Reasons for failing THV differ. Eccentric annulus calcification with incomplete stent expansion, aortic or

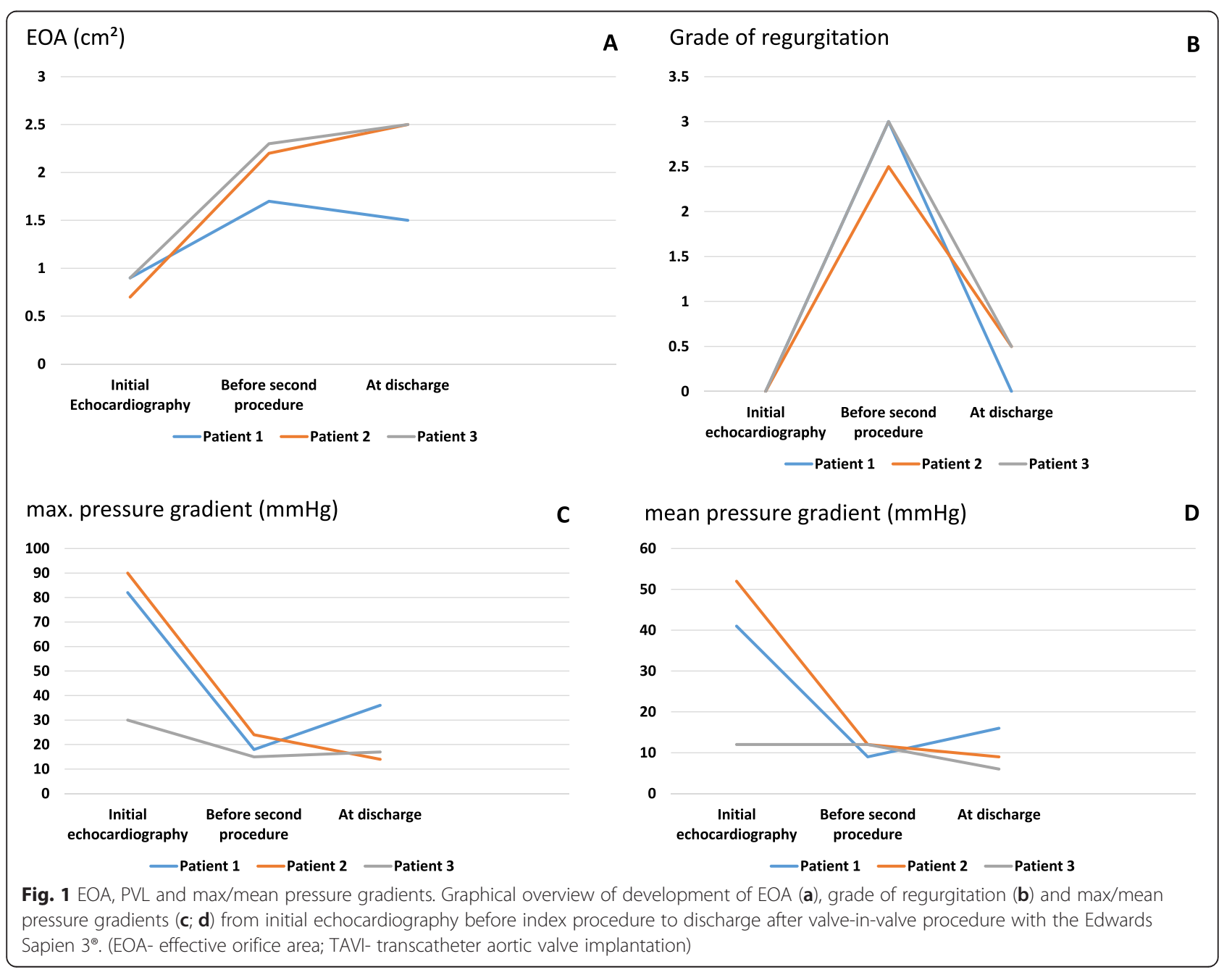


A
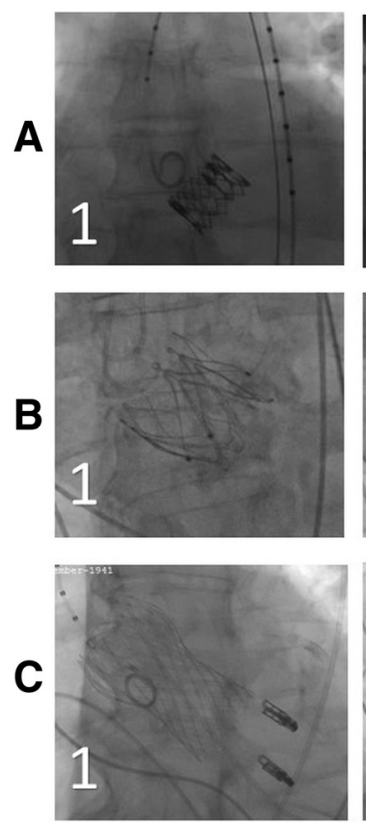
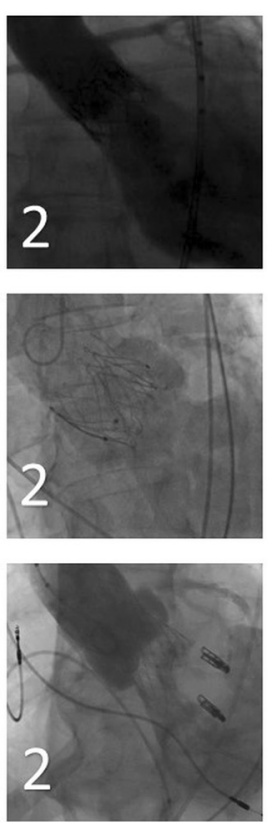
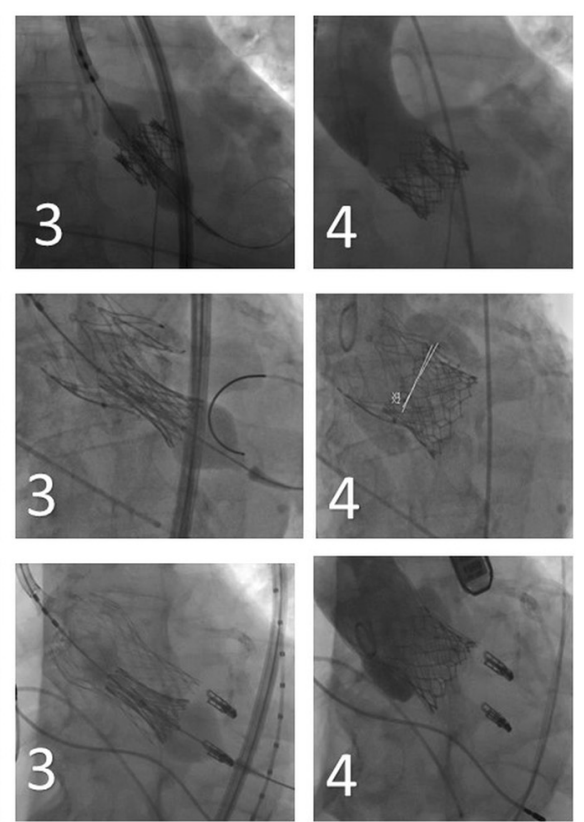

Fig. 2 Angiographies of valve-in-valve procedures with the Sapien $3^{\oplus}$ into Sapien $X T^{\oplus}$, JenaValve ${ }^{\oplus}$ and CoreValve ${ }^{\oplus}$. Column (a) with angiography of severely regurgitant Sapien XT valve without (1) and with contrast agent (2), deployment of Sapien 3 (3) and final angiography without paravalvular or transvalvular leakage (4) Column (b) presents angiography of regurgitant JenaValve without (1) and with contrast agent (2), deployment of Sapien 3 (3) and final angiography with trace paravalvular leakage and no transvalvular leakage (4) Column (c) with angiography of regurgitant CoreValve without (1) and with contrast agent (2), deployment of Sapien 3 (3) and final angiography with trace para- and no transvalvular leakage (4)

ventricular malpositioning, or malsizing of THV are among the most common causes for PVL [15-17]. Regardless of cause, PVL is responsible for inferior acute and long-term clinical outcomes.

In this work, we presented initial experience with the S3 for ViV therapy of different failing THVs in cases of relevant recurring PVL. ViV procedures were performed safely and reliably for severe PVL in the Sapien XT, the JenaValve and the CoreValve. In self-expandable THV, such as the CoreValve and the JenaValve, radial forces might be too weak in particular cases for full expansion of the stent. When suboptimal expansion occurs ballonpost-dilatation is usually performed. In our cases balloon-post-dilatation was performed at the time of the index procedure but severe PVL still recurred. PVL was increasing over a period of two to three years in these THV to an unacceptable level. As illustrated in Fig. 2, $\mathrm{ViV}$ procedures using the S3 led to further expansion of the stent of the initially implanted self-expandable THV and therefore to reduction of PVL to none/trace. Implantation of the S3 at the level of the aortic annulus was suitable and led to satisfying hemodynamic outcome.

The hemodynamic performance of the S3 in Sapien XT showed inferiority resulting in EOA of $1.5 \mathrm{~cm}^{2}$ and max/mean pressure gradient of $36 / 16 \mathrm{mmHg}$ without

Table 3 Overview of native aortic annulus, initial and second THV diameter and area

\begin{tabular}{|c|c|c|c|}
\hline & Patient no. 1 & Patient no. 2 & Patient no. 3 \\
\hline Initial annular diameter ${ }^{\mathrm{a}}(\mathrm{mm})$ & 25.3 & 26.2 & 25.6 \\
\hline Initial annular area $\left(\mathrm{mm}^{2}\right)$ & 490.3 & 620.1 & 530.7 \\
\hline Initial THV, size $(\mathrm{mm})$ & Edwards Sapien XT, 26 & JenaValve, 27 & CoreValve, 29 \\
\hline Covered annulus area of initial THV $\left(\mathrm{mm}^{2}\right)$ & $415-530$ & / & $415.5-572.6$ \\
\hline Covered annulus diameter of initial $\mathrm{THV}^{\mathrm{b}}(\mathrm{mm})$ & $23-26$ & $25-27$ & $23-27$ \\
\hline Sapien 3 size $(\mathrm{mm})$ & 26 & 29 & 29 \\
\hline Covered annulus area of implanted Sapien $3^{\mathrm{b}}\left(\mathrm{mm}^{2}\right)$ & $430-546$ & $540-683$ & $540-683$ \\
\hline Covered annulus diameter of implanted Sapien $3^{\mathrm{b}}(\mathrm{mm})$ & $23.4-26.4$ & $26.2-29.5$ & $26.2-29.5$ \\
\hline
\end{tabular}

a based on $\mathrm{CT}_{\text {eff }}=2 \times \sqrt{ }$ (circumferential area/ $\pi$ )

${ }^{b}$ according to information provided by manufacturer 
leading to clinical inferiority. This might be due to implantation of both valves at the height of the aortic annulus and/or the stout stent profile. It might be beneficial, in terms of hemodynamics, to implant THV with supraannular anchoring in those patients to avoid elevated pressure gradients and reduced EOA.

Reasons for failing of initially implanted THV, in terms of PVL, remained unclear. All patients presented only mild PVL in TTE prior to discharge after index procedure without elevated pressure gradients or inadequate EOA. Moreover all initial implanted THV covered the native aortic annulus area and diameter measured prior to the first procedure. Nevertheless, THV used for ViV procedures covered larger aortic annulus diameter and areas.

For detailed diameter and area values see Table 3.

Thus, reasons for failing are only speculative. Possibly, intra- and postprocedural underestimation of PVL in TTE and TEE examinations, stent-recoiling or measuring errors in MSCT prior to the first TAVI might have been responsible for development of severe regurgitation.

\section{Conclusions}

In conclusion, the S3 was well suitable for ViV therapy in failing THV. Adequate hemodynamic results were obtained. For further assertions concerning functional outcomes long-term results have to be awaited.

\section{Abbreviations}

CT-: Computed tomography; EuroSCORE: European System for Cardiac Operative Risk Evaluation; NYHA: New York Heart Association;

PCI: Percutaneous coronary intervention; PROM: Predicted risk of mortality; PVL: Paravalvular leakage; SAVR: surgical aortic valve replacement;

STS: Society of Thoracic Surgeons; TA: transapical; TAVI: transcatheter aortic valve implantation; TEE: Transesophageal echocardiography; TF: Transfemoral; THV: Transcatheter heart valve; TIA: transient ischemic attack;

TTE: Transthoracic echocardiography; TVL: Transvalvular leakage; VARC: Valve academic research consortium; ViV: Valve-in-valve.

\section{Competing interests}

Lenard Conradi, Hendrik Treede and Ulrich Schaefer are consultants to Edwards Lifesciences. All other authors have no potential competing interest.

\section{Authors' contributions}

AS, HT, US and LC have made substantial contributions to conception and design, acquisition of data, analysis and interpretation of data. They also have been involved in drafting the manuscript and revising it critically for important intellectual content. MS, FD, NS and YS made substantial contributions to conception and design. HR and SB has been involved in drafting the manuscript and revising it critically for important intellectual content. All authors read and approved the final manuscript.

\section{Author details}

'Department of Cardiovascular Surgery, University Heart Center Hamburg, Martinistraße 52, D-20246 Hamburg, Germany. ${ }^{2}$ Department of General and Interventional Cardiology, University Heart Center Hamburg, Martinistraße 52, Hamburg D-20246, Germany.

Received: 19 August 2015 Accepted: 10 January 2016

Published online: 15 January 2016

\section{References}

1. Smith CR, Leon MB, Mack MJ, Miller DC, Moses JW, Svensson LG, et al. Transcatheter versus surgical aortic-valve replacement in high-risk patients. N Engl J Med. 2011;364(23):2187-98.

2. Makkar RR, Fontana GP, Jilaihawi H, Kapadia S, Pichard AD, Douglas PS, et al. Transcatheter aortic-valve replacement for inoperable severe aortic stenosis. N Engl J Med. 2012;366(18):1696-704.

3. Kodali SK, Williams MR, Smith CR, Svensson LG, Webb JG, Makkar RR, et al. Two-year outcomes after transcatheter or surgical aortic-valve replacement. N Engl J Med. 2012;366(18):1686-95.

4. Seiffert M, Conradi L, Kloth B, Koschyk D, Schirmer J, Schnabel RB, et al. Single-centre experience with next-generation devices for transapical aortic valve implantation. Eur J Cardiothorac Surg. 2015;47(1):39-45.

5. Adams DH, Popma JJ, Reardon MJ, Yakubov SJ, Coselli JS, Deeb GM, et al. Transcatheter aortic-valve replacement with a self-expanding prosthesis. $\mathrm{N}$ Engl J Med. 2014;370(19):1790-8.

6. Hengstenberg C, Condado J, Garcia D, Martinez MM, La Forgia G, Ebner A, et al. TRINITY heart valve prosthesis - a novel repositionable and retrievable transapical transcatheter aortic valve system. Eurolntervention. 2015;10(11):1332-5

7. Gopal A, Grayburn PA, Mack M, Chacon I, Kim R, Montenegro D, et al. Noncontrast 3D CMR Imaging for aortic valve annulus sizing in TAVR. JACC Cardiovasc Imaging. 2015;8(3):375-8.

8. Sedaghat A, von Dobbeler C, Sontag B, Sinning JM, Fimmers R, Mellert F, et al. Use of a balloon-expandable transfemoral sheath in a TAVI cohort with complex access site - a propensity score matched analysis. Eurolntervention. 2015;11(6):698-704.

9. Vasa-Nicotera M, Sinning JM, Chin D, Lim TK, Spyt T, Jilaihawi H, et al. Impact of paravalvular leakage on outcome in patients after transcatheter aortic valve implantation. JACC Cardiovasc Interv. 2012;5(8):858-65.

10. Svensson LG, Tuzcu M, Kapadia S, Blackstone EH, Roselli EE, Gillinov AM, et al. A comprehensive review of the PARTNER trial. J Thorac Cardiovasc Surg. 2013; 145(3 Suppl):S11-6.

11. Zahn R, Schiele R, Kilkowski C, Klein B, Zeymer U, Werling C, et al. Correction of aortic regurgitation after transcatheter aortic valve implantation of the Medtronic CoreValve prosthesis due to a too-low implantation, using transcatheter repositioning. J Heart Valve Dis. 2011;20(1):64-9.

12. Eggebrecht H, Doss M, Schmermund A, Nowak B, Krissel J, Voigtländer T. Interventional options for severe aortic regurgitation after transcatheter aortic valve implantation: balloons, snares, valve-in-valve. Clin Res Cardiol. 2012;101(6):503-7.

13. Diemert $\mathrm{P}$, Lange $\mathrm{P}$, Greif $\mathrm{M}$, Seiffert $\mathrm{M}$, Conradi L, Massberg S, et al. Edwards Sapien XT valve placement as treatment option for aortic regurgitation after transfemoral CoreValve implantation: a multicenter experience. Clin Res Cardiol. 2014;103(3):183-90.

14. Shivaraju A, Ott I, Kastrati A, Kasel AM. Valve-in-valve transfemoral TAVR: Sapien 3 valve within a failed core valve bioprosthesis. Eur Heart J. 2014;7;35(38):2684

15. Gripari $P$, Ewe $S H$, Fusini $L$, Muratori $M, N g A C$, Cefalù $C$, et al. Intraoperative 2D and 3D transoesophageal echocardiographic predictors of aortic regurgitation after transcatheter aortic valve implantation. Heart. 2012;98(16):1229-36.

16. Buzzatti N, Maisano F, Latib A, Cioni M, Taramasso M, Mussardo M, et al. Computed tomography-based evaluation of aortic annulus, prosthesis size and impact on early residual aortic regurgitation after transcatheter aortic valve implantation. Eur J Cardiothorac Surg. 2013;43(1):43-50.

17. Willson AB, Webb JG, Labounty TM, Achenbach S, Moss R, Wheeler M, et al. 3-dimensional aortic annular assessment by multidetector computed tomography predicts moderate or severe paravalvular regurgitation after transcatheter aortic valve replacement: a multicenter retrospective analysis. J Am Coll Cardiol. 2012;59(14):1287-94. 